\title{
Prevenção de intercorrências estomatológicas em oncologia pediátrica
} Prevention of stomatological complications in pediatric oncology

\author{
Isabel Nemoto Vergara Sasada* \\ Claudia Marcela Hernández Cancino* \\ Renata Córdova Petersen ${ }^{* * *}$ \\ Ingeburg Hellwig* \\ Caroline Siviero Dillenburg****
}

\section{Resumo}

Objetivo: este artigo visa demonstrar por meio da Revisão de literatura e do relato de dois casos a importância da aplicação de protocolos de adequação do meio bucal em crianças submetidas a tratamento oncológico. As neoplasias em crianças e adolescentes estão entre $1 \%$ e $3 \%$ de todos os tumores malignos na maioria da população. Representam a principal causa de morte a partir dos 5 anos de idade. Para tratar essas doenças, os pacientes são submetidos à quimioterapia e à radioterapia, tratamentos que podem ocasionar alterações na integridade e na função dos tecidos da cavidade bucal. A toxicidade das drogas quimioterápicas pode ser direta ou indireta, aguda ou tardia, causando impacto na saúde e na qualidade de vida dos pacientes. Relato de casos: neste artigo são relatados dois casos de crianças diagnosticadas com neoplasias. Em uma delas, foi realizada a adequação do meio bucal antes do tratamento oncológico para prevenir intercorrências estomaológicas e, na outra, cujas condições de saúde bucal eram favoráveis, mas que desenvolveu mucosite durante o tratamento oncológico, foi instituído um protocolo para tratar as lesões. Considerações finais: as inadequadas condições de saúde bucal prévias ao tratamento oncológico influenciam diretamente nas intercorrências estomatológicas, aumentando os riscos de infecção local e sistêmica, prejudicando a alimentação e a recuperação do paciente e, dessa forma, aumentando o uso de analgésicos e de dias de internação hospitalar. A avaliação, o tratamento odontológico e o condicionamento bucal, previamente ao tratamento oncológico, tem sido importante na prevenção de sequelas para os pacientes pediátricos.

Palavras-chave: Estomatologia. Quimioterapia. Radioterapia. Saúde bucal.

\section{Introdução}

A evolução dos protocolos quimioterápico e radioterápico no tratamento das neoplasias pediátricas tem determinado um aumento progressivo nos índices de cura. As estatísticas mostram que nos anos 1960 a sobrevida que era de apenas 4\% alcançou níveis acima de $70 \%$ em 2012, desde que o diagnóstico seja precoce e o tratamento seja realizado em centros especializados ${ }^{1}$. Dados de literatura mostram que cerca de $40 \%$ dos pacientes oncológicos apresentam complicações bucais agudas decorrentes da toxicidade direta ou indireta, como mucosite, xerostomia, infecções fúngicas, virais ou bacterianas ${ }^{2}$. Os efeitos adversos envolvendo a cavidade bucal e a sua severidade estão relacionados à quimioterapia, à radioterapia, ao tipo e à localização do tumor e, ainda, às condições de saúde bucal. Os pacientes com condições de saúde bucal desfavoráveis, infecções dentárias, gengivites e falta de higiene bucal, apresentam um risco maior de desenvolver complicações que podem comprometer as funções sistêmicas durante os períodos de imunossupressão induzidos pela quimioterapia. A radioterapia na região de cabeça e pescoço tende a potencializar esses efeitos colaterais.

Entre os efeitos tardios do tratamento oncológico, observa-se que as alterações odontológicas e do desenvolvimento facial são sequelas do tratamento oncológico e tem maior impacto, pois as crianças vivem por um período maior de tempo, necessi-

Mestre em Saúde da Criança e do Adolescente pela Faculdade de Medicina da Universidade Federal do Rio Grande do Sul e Coordenadora do Curso de Especialização em Odontologia para Pacientes com Necessidades Especiais ABO-RS, Porto Alegre, RS, Brasil.

Doutora em Cirurgia e Traumatologia Bucomaxilofacial pela Faculdade de Odontologia da Pontifícia Universidade Católica do Rio Grande do Sul e Professora do Curso de Especialização em Odontologia para Pacientes com Necessidades Especiais ABO-RS, Porto Alegre, RS, Brasil.

Especialista em Ortodontia pela Sociedade Brasileira de Cirurgiões Dentistas e aluna do Curso de Especialização em Odontologia para Pacientes com Necessidades Especiais ABO-RS, Porto Alegre, RS, Brasil.

**** Mestre em Patologia Oral pela Universidade Federal do Rio Grande do Sul e Professora do Curso de Especialização em Odontologia para Pacientes com Necessidades Especiais ABO-RS, Porto Alegre, RS, Brasil. 
tando, portanto, de cuidados preventivos ${ }^{3}$. Quanto mais jovem for o paciente, maior é a possibilidade das terapias oncológicas afetarem a saúde bucal e o desenvolvimento dentário e facial. As manifestações dos efeitos colaterais em crianças menores de 12 anos são duas vezes maiores que em pacientes adultos. Essa diferença parece estar associada ao índice mitótico elevado das células da mucosa bucal e ao desenvolvimento dentário nessa faixa etária ${ }^{4,5}$.

Este artigo tem por objetivo realizar uma revisão da literatura a respeito dos protocolos de adequação do meio bucal utilizados em crianças submetidas a tratamento oncológico e apresentar dois casos de pacientes pediátricos e os protocolos aplicados.

\section{Revisão de literatura}

O primeiro relato de lesões bucais decorrentes do tratamento oncológico foi realizado por Del Regato $^{5}$ (1939) relacionando à xerostomia e ao surgimento de cáries à radioterapia. As cáries de radiação apresentam uma evolução muito rápida e podem levar à destruição dentária. A preocupação com as manifestações bucais de toxicidade dos agentes quimioterápicos está relacionada com o risco aumentado de infecção sistêmica, a dificuldade na alimentação e a dor.

O tratamento oncológico, geralmente, inclui a utilização de quimioterapia e radioterapia que podem provocar toxicidade em diversos órgãos, incluindo a cavidade bucal, especialmente no paciente pediátrico. Essas alterações podem ser locais e imediatas, como ocorre em casos de tumores na região de cabeça e de pescoço tratados com radioterapia, ou podem ser difusas nos casos de pacientes que recebem quimioterapia e radioterapia. A toxicidade direta e indireta dos agentes antineoplásicos pode causar dor, mucosite, dificuldades para mastigação e deglutição, disfunções glandulares e alterações no paladar. Essas condições podem comprometer o estado nutricional do paciente, prejudicando a recuperação, a evolução do tratamento e, muitas vezes, causando o atraso nos ciclos subsequentes de quimioterapia ou nas sessões de radioterapia ${ }^{6}$.

$\mathrm{O}$ conhecimento de que algumas drogas quimioterápicas provocam maior toxicidade para mucosa bucal permite aplicar medidas preventivas. Medicações como o Metotrexato, Ciclofosfamida e a Doxorrubicina são as mais envolvidas com o surgimento da mucosite oral em crianças ${ }^{7}$. Recomenda-se que, previamente ao início do tratamento oncológico, o paciente seja examinado para identificação das possíveis doenças bucais ${ }^{6}$, incluindo aquelas latentes que podem exacerbar-se nos períodos de imunossupressão.

Durante o tratamento antineoplásico, as complicações bucais devem ser prevenidas e tratadas. Medidas preventivas, tais como o uso tópico de di- gluconato de clorexidina a $0,12 \%$, a remoção de placa e a intensificação dos cuidados de higiene bucal devem ser incentivados. A prescrição de flúor tópico, sob forma de gel ou bochechos é fundamental para prevenção de problemas odontológicos ${ }^{8,9}$. Toth et al. ${ }^{10}$ (1995) reforçam a ideia de que a integridade da boca deve ser garantida pelos cuidados iniciais, antes que a terapia oncológica comprometa os tecidos dessa região anatômica e antes que o desconforto do paciente, a dificuldade para falar e para se alimentar constituam-se como realidade.

Mucosite oral é a inflamação da mucosa bucal e pode resultar da ação dos agentes quimioterápicos, variando de eritema até ulceração, sendo agravada pela inadequada saúde bucal. É um processo complexo, envolvendo todos os tecidos e elementos celulares da mucosa. Novas evidências sugerem que o risco de desenvolvimento da mucosite pode ser determinado por fatores genéticos ${ }^{11}$. $\mathrm{O}$ dano mucoso secundário ao tratamento antineoplásico é, igualmente, complexo e parece surgir de uma série de eventos celulares e moleculares que ocorrem não só em epitélio, mas, também, no estroma subjacente ${ }^{12}$. Diferenças genéticas na taxa de apoptose tecidual, lesões microvasculares, a partir da apoptose do endotélio, e taxas sanguíneas periféricas aumentadas para o fator de necrose tumoral alfa e interleucina- 6 parecem estar envolvidas. Além dos efeitos diretos dos agentes antineoplásicos, fatores de risco adicionais incluem a pouca idade, o gênero feminino, a higiene bucal deficiente, os focos de infecção bucal, a má nutrição, a função salivar deficiente, o uso de tabaco e/ou de álcool.

A toxicidade dos quimioterápicos, que inclui a imunossupressão do paciente, associada à alteração do fluxo salivar e às lesões da mucosa, contribui para aumentar o risco de infecções oportunistas. Essas infecções podem ser de etiologia fúngica, viral ou bacteriana ${ }^{13}$. A candidíase é a infecção mais frequente nesses pacientes, pode invadir os tecidos locais, estendendo-se ao esôfago e aos pulmões, produzindo septicemia e em situações graves óbito. Em pacientes neutropênicos, a terapia sistêmica antifúngica é recomendada para que se obtenha maior eficiência no combate a essa infecção ${ }^{4}$.

As infecções virais por herpes simples, herpes zoster, citomegalovirus e Epstein Barr vem em segundo lugar, nesses casos, também é recomendada a terapia sistêmica para obtenção de maior eficiência. As infecções bacterianas de origem dentária e periodontal são frequentes nos períodos de neutropenia. Com a redução do fluxo salivar, há um desequilíbrio da flora bacteriana e um aumento da atividade de cárie e de doenças periodontais ${ }^{14}$. Todo processo infeccioso que tem origem na cavidade bucal apresenta grande risco de evoluir para contaminação sistêmica e, por isso, é extremamente importante que sejam instituídos os cuidados de higiene, a prevenção e o tratamento prévio de qualquer alteração bucal. 
A toxicidade da quimioterapia e os efeitos da radioterapia na região da cabeça e do pescoço podem levar à xerostomia temporária ou permanente. Ocorre uma alteração quantitativa e qualitativa do fluxo salivar com perda das propriedades físico químicas da saliva, causando dificuldade de mastigação deglutição, alteração do paladar, aumento do número de cáries e da velocidade da destruição dentária ${ }^{15}$. O fluxo salivar é necessário para a saúde bucal, pois as glicoproteinas da saliva diminuem a permeabilidade da mucosa e as enzimas possuem atividade antimicrobiana ${ }^{16}$. A xerostomia aumenta o risco de infecções na cavidade bucal, bem como de traumatismos e de contaminação sistêmica ${ }^{11}$.

As hemorragias gengivais são causadas pela ulceração do epitélio do sulco gengival e pela necrose do tecido subjacente, que em períodos de plaquetopenia são frequentes e prejudicam, consideravelmente, as manobras de higiene bucal aumentando o risco de infecções ${ }^{17}$. Sempre que houver a necessidade de interrupção da escovação dentária e do uso de fio dental é necessário que se introduzam alternativas para a manutenção da higiene. Soluções antissépticas para bochechos mais frequentes, o uso de gaze sobre as superfícies dentárias e escovas especiais são úteis e são alternativa a serem consideradas.

$\mathrm{Na}$ radioterapia de cabeça e pescoço, a ação direta da radiação sobre as papilas gustativas pode levar à ageusia (perda) ou disgeusia (alteração do paladar), que, associada à xerostomia e à mucosite, assume uma gravidade maior, pois a nutrição do paciente fica prejudicada e a recuperação mais lenta ${ }^{6}$.

A mucosite, geralmente, surge entre 7 e 10 dias após o início da quimioterapia e sua severidade depende da agressividade da droga, doses altas e duração prolongada do tratamento utilizado. As mucosites são autolimitadas quando não complicadas por infecções e, usualmente, desaparecem entre $2 \mathrm{e}$ 4 semanas após cessar o efeito citotóxico da quimioterapia ${ }^{13}$. A manifestação mais precoce na mucosa é o desenvolvimento de uma coloração esbranquiçada pela ausência de descamação suficiente da ceratina. Isso logo é seguido pela perda dessa camada e substituição pela mucosa atrófica, a qual é edemaciada, eritematosa e friável. Subsequentemente, aparecem áreas de ulceração com a formação de uma membrana superficial fibrinopurulenta amarelada e removível ${ }^{3}$.

A dor, a ardência e o desconforto são sintomas que podem se acentuar durante a alimentação ou a higiene bucal $^{8}$. A classificação do grau de mucosite segundo a Organização Mundial de Saúde (OMS) foi definida como: grau 0 - mucosa normal, íntegra; grau 1- irritação, dor e eritema; grau 2 - eritema, úlcera, permitindo alimentação sólida; grau 3 - úlceras e dieta líquida; grau 4 - impossível alimentação via oral (VO).

Köstler et al. ${ }^{18}$ (2001) relataram que em pacientes em tratamento com quimioterapia, a mucosa não queratinizada do palato mole, bochechas e lábios, a superfície ventral da língua e o assoalho da boca são as áreas mais vulneráveis à toxicidade direta. Já a mucosa queratinizada da gengiva, do dorso da língua e do palato duro é mais raramente afetada, provavelmente devido à sua menor renovação celular.

\section{Relato de casos}

Criança do gênero masculino com 8 anos e 5 meses de idade e diagnóstico de meduloblastoma. O tratamento proposto foi cirurgia, quimioterapia e radioterapia do crânio e neuro-eixo. $\mathrm{O}$ paciente apresentava condições de saúde bucal desfavoráveis com presença de cáries profundas e comprometimento pulpar na maioria dos dentes (Figuras 1 e 2). Foram realizadas exodontias, restaurações e tratamento profilático preventivo em tempo único, sob anestesia geral, antes de iniciar tratamento oncológico. Paciente respondeu bem ao tratamento do Meduloblastoma e não apresentou quadros graves de Mucosite.

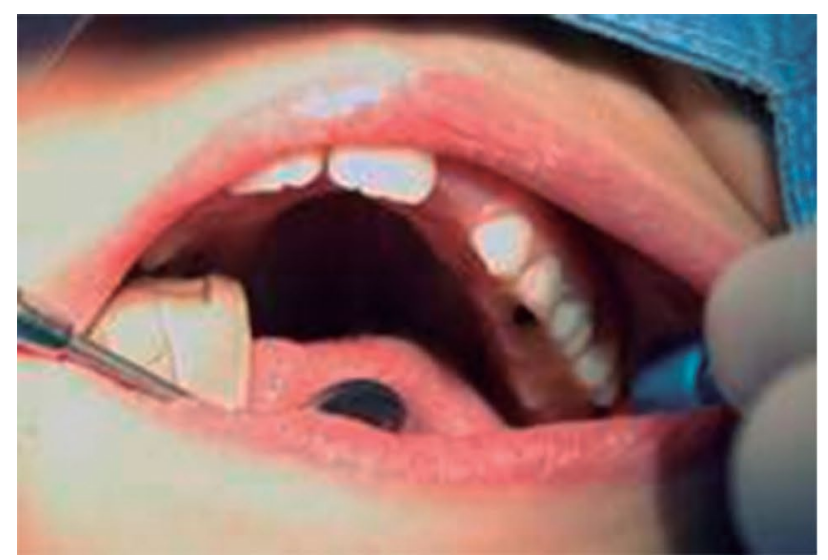

Figura 1 - Paciente com lesões cariosas

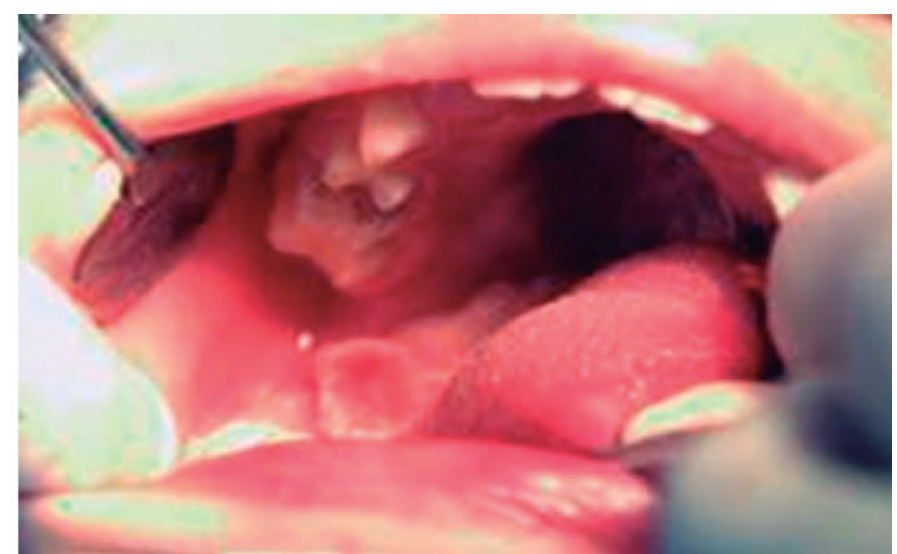

Figura 2 - Lesões cariosas 
Paciente do gênero feminino com 15 anos e 3 meses de idade, diagnóstico de Osteossarcoma em tíbia, sem metástases. O tratamento proposto foi cirurgia e quimioterapia com alto risco de toxicidade bucal. As condições de saúde bucal eram favoráveis e a paciente colaborativa. Desenvolveu mucosite de grau 4 após o primeiro curso de quimioterapia com metotrexato, apresentando úlceras extensas na mucosa jugal, ventre e bordas da língua, palato duro e crostas hemáticas em lábios (Figuras 3 e 4). Além da analgesia sistêmica e dos bochechos com clorexidine duas vezes ao dia, foi aplicado laser de baixa potência no sítio das lesões. Após 14 dias de laserterapia, a paciente apresentou recuperação total da mucosite. Os níveis séricos de metotrexato estavam acima do observado usualmente. Nos cursos subsequentes de quimioterapia, foram intensificados os cuidados preventivos. A paciente voltou a apresentar complicações orais, porém de menor intensidade.

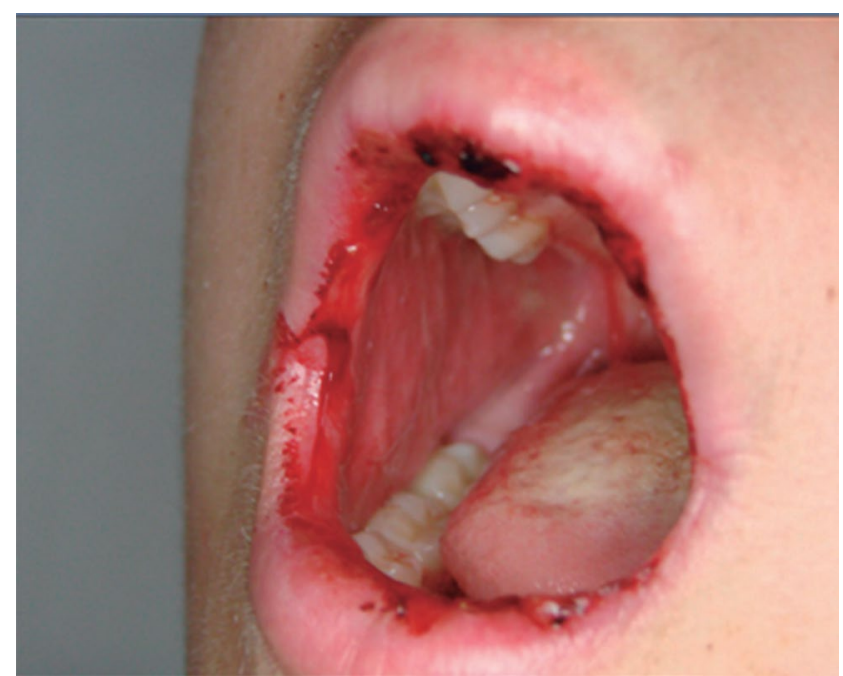

Figura 3 - Mucosite - Lesões na mucosa jugal e na língua

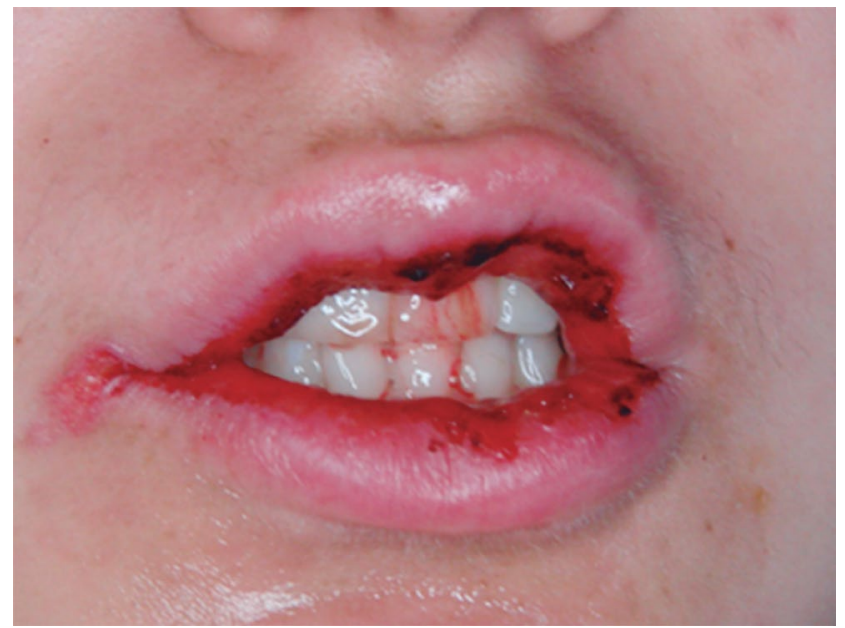

Figura 4 - Mucosite - Lesões nos lábios

\section{Discussão}

A toxicidade dos quimioterápicos, que inclui a imunossupressão do paciente, associada à alteração do fluxo salivar e às lesões da mucosa contribuem para aumentar o risco de aquisição de infecções oportunistas. Essas infecções podem ser de etiologia fúngicas, virais ou bacterianas ${ }^{15}$. A candidíase, infecção fúngica determinada pela Cândida Albicans, é a que mais acomete esses pacientes e pode se proliferar, invadindo os tecidos locais, estendendo-se ao esôfago e aos pulmões, produzindo septicemia e, em situações extremas determinar óbito. Em pacientes neutropênicos, a terapia sistêmica antifúngica é recomendada para que se obtenha maior eficiência no combate a essa infecção ${ }^{4}$. As infecções virais por herpes simples, herpes zoster, citomegalovirus e Epstein Barr vem em segundo lugar e também é recomendada a terapia sistêmica para obtenção de maior eficiência. As infecções bacterianas de origem dentária e periodontal são frequentes nos períodos de neutropenia. Com a redução do fluxo salivar, há um desequilíbrio da flora bacteriana e um aumento da atividade de cárie e doenças periodontais ${ }^{13}$. Todo processo infeccioso que tem origem na cavidade bucal apresenta grande risco de evoluir para contaminação sistêmica, prejudicando a saúde do paciente e a recuperação, por isso é extremamente importante que protocolos de higiene, de prevenção e de tratamento prévio sejam implantados.

\section{Considerações finais}

O tratamento antineoplásico, que inclui quimioterapia e radioterapia, atua na divisão celular e, a cavidade bucal, pelo alto índice de renovação celular, é muito suscetível. As condições de saúde bucal são fatores etiológicos determinantes das intercorrências estomatológicas. Os pacientes oncológicos necessitam de protocolos de atenção especial, pois, em períodos de neutropenia, estão mais suscetíveis às infecções bucais, hemorragias, mucosites e reagudização de processos crônicos. A higiene rigorosa é fundamental para a manutenção da saúde bucal e para sua recuperação frente às intercorrências estomatológicas. A inclusão da avaliação estomatologica e do tratamento odontológico, de forma previa ao planejamento quimioterápico e/ou radioterápico, principalmente na região cérvico-facial, auxiliará na prevenção de complicações sistêmicas e locais que possam inviabilizar o seguimento adequado do tratamento antineoplásico proposto. 


\section{Abstract}

Objective: this article aims to show, through literature review and the report of two cases, the importance of applying protocols of adequacy of oral environment to children undergoing cancer treatment. Neoplasia in children and adolescents represent from $1 \%$ and $3 \%$ of all malignant tumors in the majority of the population. It is the main cause of death of children from five years old and up. The treatment consists in submitting patients to chemotherapy and radiotherapy, which may lead to changes in the integrity and function of oral cavity tissues. The toxicity of chemotherapy drugs may be direct or indirect, acute or delayed, and may cause an impact on health and the quality of life of patients. Case reports: this article reports two cases of children diagnosed with neoplasias. One of them was treated with the adequacy of oral environment before cancer treatment to prevent stomatological complications, and the other child, whose oral health conditions were favorable but presented mucositis during cancer treatment, a protocol to treat the injury was established. Final considerations: the inadequate oral health conditions prior to cancer treatment directly influence stomatological complications, increasing the risk of local and systemic infection, impairing feeding and recovery of the patient, and thus increasing the use of analgesics and hospitalization time. Assessment, dental treatment, and oral condition prior to cancer treatment have been important in preventing sequelae in pediatric patients.

Keywords: Stomatology; Chemotherapy; Radiotherapy; Oral health.

\section{Referências}

1. Estimativa 2012 - Incidencia de Câncer no Brasil - INCA MS. Coordenação Geral de Ações Estrategicas. Coordenação de Açaõ e Vigilãncia. Rio de Janeiro: Inca; 2012.

2. Martins ACM, Caçador NP, Gaeti WP. Complicações bucais da quimioterapia antineoplásica. Acta Scientiarum 2002;24(3):663-70.

3. Maciel JC, de Castro CG, Jr., Brunetto AL, Di Leone LP, da Silveira HE. Oral health and dental anomalies in patients treated for leukemia in childhood and adolescence. Pediatr Blood Cancer 2009;53(3):361-5.

4. Sonis ST, Fazio RC, Fang L. Princípios e Prática de Medicina Oral. 2. ed. Rio de Janeiro: Guanabara Koogan; 1996.

5. Del Regato JA. Dental lesions after roentgen therapy in câncer of the buccal cavity, pharynx and larynx. Am j Roentgenol Radiat Ther Nuclear Med 1939;42(3):404-10.

6. Rosa LN. Atenção estomatológica aos pacientes pediátricos oncológicos. Rev Gaúcha odontologica 1997;45(2):111-14.

7. Hespanhol FL. Levantamento epidemiológico de manifestações bucais em pacientes submetidos a quimioterapia [Dissertação de Mestrado]. Duque de caxias: Universidade do Grande Rio Professor José de Souza Herdy. Escola de Odontologia; 2007b.

8. Santos PSS, Messaggi AC, Mantesso A, Magalhães Santos MHC. Mucosite oral: perspectivas atuais na prevenção e tratamento Rev Gaúcha Odontol 2009;57(3):339-44.
9. Santos PSS. Avaliação da mucosite oral em pacientes que receberam adequação bucal prévia ao transplante de medula óssea [Dissertação de Mestrado]. São Paulo: Faculdade de Odontologia da Universidade de São Paulo; 2005.

10. Toth BB, Chambers MS, Fleming TJ, Lemon JC, Martin JW. Section of Oncologic Dentistry and Prosthodontics 1995;9(9):851-8.

11. Sonis ST, Elting LS, Keefe D, Peterson DE. Perspectives on cancer therapy - induced mucosal injury: pathogenesis, measurement, epidemiology, and consequences for patients. Cancer 2004;100(9):1995-2025.

12. Neville BW, Damm DD, Allen CM, Bouquot JE. Patologia Oral e Maxilofacial. 3. ed. Rio de Janeiro: Elsevier; 2009.

13. Albuquerque RA, Morais VL, Sobral APV. Protocolo de atendimento odontológico a pacientes oncológicos pediátricos revisão da literatura. Rev Odontol Unesp 2007;36(3):275-80.

14. Kroetz FM, Czlusniak G D. Alterações bucais e condutas terapéuticas em pacientes infanto-juvenis submetidos a tratamentos anti-neoplásicos. Public Uepg Biol Health Sci 2003;9(2):41-8.

15. Dib LL, Gonçalves RCC, Kowalski LP, Salvajoli JV. Abordagem multidisciplinar das complicações orais da radioterapia. Rev Assoc Paul Cir Dentistas 2000;54(5):391-6.

16. McCarthy GM, Awde JD, Ghandi H, Vincent M, KochaRisk WI. Factors associated with mucositis in cancer patients receiving 5-fluorouracil. Oral Oncology 1998;34(6):484-90.

17. McKenna, SJ. Leukemia. Oral Surg Oral Med Oral Pathol Oral Radiol Endod 2000;89(2):173-9.

18. Köstler WJ, Hejna M, Wenzel C, Zielinski CC. Oral Mucositis Complication Chemoterapy and/or Radiotherapy: options for prevention and treatment. CA Cancer J Clin 2001;51(5):290315

\section{Endereço para correspondência:}

Isabel Nemoto Vergara Sasada

Rua Furriel Luiz Antonio de Vargas, 134

904701130 Porto Alegre RS

Telefone: (51) 8124-7238/ 3330-1597/ 3395-3704

Email: isanemoto@yahoo.com.br

Recebido: 16/03/2015. Aceito: 28/04/2015. 